\title{
Comorbidades em mato-grossenses hospitalizados com COVID-19 em 2020
}

\author{
Fernanda Rocha Anjos de Oliveira \\ Mestranda do Programa de pós-graduação em Saúde Coletiva, Universidade Federal de Mato Grosso (UFMT), campus de \\ Cuiabá, Mato Grosso, Brasil. \\ $\triangle$ fernandarochaanjos@gmail.com \\ Thalia Baldissarelli Cunha \\ Aluna de Iniciação Científica do curso de Enfermagem na Universidade do Estado de Mato Grosso (UNEMAT), campus de \\ Tangará da Serra, Mato Grosso, Brasil. \\ $\triangle$ thaliabaldissarelli@hotmail.com \\ Juliana Herrero da Silva \\ Mestre em Genética e Biologia Molecular. Prefeitura Municipal de Tangará da Serra, Mato Grosso, Brasil. \\ $\bowtie$ julianaherrerodasilva@hotmail.com

\section{Marina Atanaka} \\ Doutora em Saúde Pública. Docente do Programa de pós-graduação em Saúde Coletiva, Universidade Federal de Mato \\ Grosso, campus de Cuiabá, Mato Grosso, Brasil. \\ $\triangle$ marina.atanaka@gmail.com

\section{Vagner Ferreira do Nascimento} \\ Doutor em Bioética. Professor na Universidade do Estado de Mato Grosso (UNEMAT), campus de Tangará da Serra, Mato \\ Grosso, Brasil. \\ $\triangle$ vagnerschon@hotmail.com
}

\section{Ana Cláudia Pereira Terças-Trettel}

Doutora em Medicina Tropical. Professora na Universidade do Estado de Mato Grosso (UNEMAT), campus de Tangará da Serra, Mato Grosso, Brasil e Programa de pós-graduação em Saúde Coletiva, Universidade Federal de Mato Grosso, campus de Cuiabá, Mato Grosso, Brasil.

$\triangle$ ana.claudia@unemat.br

Recebido em 22 de março de 2021

Aceito em 9 de novembro de 2021

\section{Resumo:}

Introdução: A pandemia da COVID-19 trouxe consigo a emergência de uma doença infecciosa que afeta especificamente o aparelho respiratório, que varia de quadros leves a graves, principalmente em indivíduos portadores de comorbidades pré-existentes. O objetivo deste estudo foi caracterizar as comorbidades em matogrossenses hospitalizados com COVID-19. Metodologia: Trata-se de um estudo observacional, ecológico e com abordagem quantitativa. A coleta de dados foi realizada diretamente no sistema de informação da vigilância epidemiológica, cujos dados foram duplamente tabulados e realizada análise descritiva das variáveis estudadas. Resultados: Predominou neste estudo população composta por homens (61,29\%), idosos (59,14\%), autodeclarados pardos (52,69\%), que eram usuários do Sistema Único de Saúde (SUS) (65,59\%). Entre os pacientes internados, 76,34\% relataram ser portadores de comorbidades pré-existentes à internação, sendo as mais prevalentes Hipertensão Arterial Sistêmica (HAS), Diabetes Melittus (DM) e doenças renais. Discussão: Verificouse que o fator idade e sexo influenciam na incidência de casos novos da doença, e que possuir comorbidades é fator de risco para desenvolvimento de complicações e casos graves da doença. A grande prevalência de comorbidades influencia no aumento de hospitalizações por COVID-19 o que tende a sobrecarregar o sistema de saúde, bem como o desfecho clínico dos pacientes hospitalizados. Este estudo permite o conhecimento do perfil de saúde e das vulnerabilidades vivenciadas por esta população, bem como favorece a intervenção efetiva na promoção de saúde e prevenção de agravos relacionados à incidência da COVID-19 concomitante às condições crônicas de saúde.

Palavras-chave: COVID-19, doenças crônicas, internação hospitalar, SUS. 


\title{
Comorbidities in Mato Grosso residents hospitalized with COVID-19 in 2020
}

\begin{abstract}
:
Introduction: The COVID-19 pandemic brought with it the emergence of an infectious disease that specifically affects the respiratory system, ranging from mild to severe, especially in individuals with pre-existing comorbidities. The aim of this study was to characterize comorbidities in Mato Grosso hospitalized with COVID-19. Methodology: This is an observational, ecological study with a quantitative approach. Data collection was performed directly in the epidemiological surveillance information system, whose data were doubly tabulated and a descriptive analysis of the studied variables was performed. Results: In this study, a population composed of men (61.29\%), elderly (59.14\%), self-declared browns (52.69\%), who were users of the Unified Health System (SUS) (65.59\%) predominated. Among hospitalized patients, $76.34 \%$ reported having pre-existing comorbidities at admission, the most prevalent being Systemic Arterial Hypertension (SAH), Diabetes Melittus (DM) and kidney diseases. Discussion: It was found that the factor age and sex influence the incidence of new cases of the disease, and that having comorbidities is a risk factor for the development of complications and severe cases of the disease. The high prevalence of comorbidities influences the increase in hospitalizations for COVID-19, which tends to overburden the health system, as well as the clinical outcome of hospitalized patients. This study allows the knowledge of the health profile and the vulnerabilities experienced by this population, as well as favors the effective intervention in health promotion and prevention of diseases related to the incidence of COVID-19 concomitant with chronic health conditions.
\end{abstract}

Keywords: COVID-19, chronic diseases, hospitalization, SUS.

\section{Comorbilidades en residentes de Mato Grosso hospitalizados con COVID-19 en 2020}

\section{Resumen:}

Introducción: La pandemia de COVID-19 trajo consigo la aparición de una enfermedad infecciosa que afecta específicamente al sistema respiratorio, de leve a grave, especialmente en individuos con comorbilidades preexistentes. El objetivo de este estudio fue caracterizar las comorbilidades en Mato Grosso hospitalizados con COVID-19. Metodología: Se trata de un estudio ecológico observacional con enfoque cuantitativo. La recolección de datos se realizó directamente en el sistema de información de vigilancia epidemiológica, cuyos datos fueron doblemente tabulados y se realizó un análisis descriptivo de las variables estudiadas. Resultados: En este estudio predominó una población compuesta por hombres $(61,29 \%)$, ancianos $(59,14 \%)$, marrones autodeclarados $(52,69 \%)$, que eran usuarios del Sistema Único de Salud (SUS) (65,59\%). Entre los pacientes hospitalizados, el 76,34\% refirió tener comorbilidades preexistentes al ingreso, siendo las más prevalentes la Hipertensión Arterial Sistémica (HSA), lo diabetes Melittus (DM) y las enfermedades renales. Discusión: Se encontró que el factor edad y sexo influyen en la incidencia de nuevos casos de la enfermedad, y que tener comorbilidades es un factor de riesgo para el desarrollo de complicaciones y casos severos de la enfermedad. La alta prevalencia de comorbilidades influye en el aumento de las hospitalizaciones por COVID-19, que tiende a sobrecargar el sistema de salud, así como el resultado clínico de los pacientes hospitalizados. Este estudio permite conocer el perfil de salud y las vulnerabilidades que vive esta población, así como favorece la intervención efectiva en la promoción de la salud y prevención de enfermedades relacionadas con la incidencia de COVID-19 concomitante con condiciones crónicas de salud.

Palabras clave: COVID-19, enfermedades crónicas, hospitalización, SUS. 


\section{INTRODUÇÃo}

A COVID-19 é uma doença respiratória aguda grave, causada pelo novo coronavírus (SARS-CoV-2) descoberto em dezembro de 2019, em Wuhan, na China. A COVID-19 se tornou uma pandemia, devido às características adaptativas do SARS-COV-2 que facilitam sua transmissão, assim como em razão do comportamento da população e governos frente às medidas de controle e proteção (Li et al., 2020).

Esta pandemia alterou a rotina e vida das pessoas em todo mundo e impôs novas formas de relacionamento. Isso trouxe sofrimentos, quer seja pela frustração ou dificuldade no processo de adaptação como pelos danos gerados nas circunstâncias de desemprego (TEIXEIRA, 2020), distanciamento da família (AMMAR et al., 2020), risco de infecção (CARRATURO, 2020), diagnóstico da COVID-19 (TALEGHANI, 2020) e óbito (CALÓ et al., 2020).

Esses sofrimentos podem ser ainda maiores em pessoas que integram grupos de risco, pois além de vivenciarem as restrições pandêmicas, devem possuir cuidados extras, dada às suas condições clínicas e históricos de saúde que ampliam a vulnerabilidade para desfechos desfavoráveis na ocorrência da COVID-19 (HU et al., 2020). As pessoas com comorbidades, após infectadas podem apresentar quadros clínicos mais complexos e diversificados, com riscos aumentados de complicações que contribuem para hospitalizações que em sua maioria podem conduzir ao óbito (EJAZ et al., 2020). Logo, pessoas com comorbidades possuem mais chances de desenvolverem formas graves nas infecções pelo SARS-CoV-2 (BRASIL, 2020).

À nível nacional, entre aqueles com síndrome respiratória aguda grave por COVID-19, as comorbidades mais predominantes foram diabetes mellitus, doença cardiovascular, doença renal crônica e pneumopatias crônicas (NIQUINI et al., 2020). Além disso, autores também apontam as dislipidemias e a obesidade como fatores que aumentam os riscos para o agravamento do quadro clínico (CALÓ et al., 2020).

No Brasil, a região Centro Oeste possui a maior prevalência de doenças cardiovasculares e obesidade masculina, e diabetes entre mulheres maiores de 50 anos (NUNES et al., 2020). Especificamente no Estado de Mato Grosso, entre os óbitos por COVID19, a maioria dos indivíduos possuía mais de uma doença pré-existente (CALÓ et al., 2020). 
Esse cenário motivou aprofundar sobre as comorbidades em pacientes com COVID-19 no contexto mato-grossense, na perspectiva de elucidar características para o direcionamento clínicoassistencial. Dessa forma, o objetivo do estudo foi caracterizar as comorbidades em mato-grossenses hospitalizados com COVID-19.

\section{METODOLOGIA}

Trata-se de estudo observacional, ecológico e com abordagem quantitativa. A coleta de dados foi realizada diretamente no sistema de informação da vigilância epidemiológica do maior município da região médio norte de Mato Grosso, Brasil. A escolha desse município ocorreu por sediar uma regional de saúde e ser referência para internação de COVID-19.

Para a coleta, utilizou-se um roteiro criado pelos próprios pesquisadores, com variáveis disponíveis nesse sistema (sexo, faixa etária, cor, categoria profissional, tipo de comorbidade, tipo de instituição de internação), filtrando o período do segundo e terceiro trimestre da pandemia da COVID19, correspondente aos meses de junho a novembro de 2020. A escolha desse recorte deve-se as importantes oscilações da média móvel de novos casos e óbitos pela COVID-19 no Brasil nesse período.

Após o término da coleta de dados, realizou-se a digitação dupla dos dados em planilha do Microsoft Excel ${ }^{\circledR}$, que posteriormente foram confrontados, para monitorar possíveis inconsistências ou erros. Na sequência, o banco de dados foi importado para o software SPSS versão $26.0 \mathrm{com}$ realização de análise descritiva simples através do programa Excel $^{\circledR}$ das variáveis sociodemográficas (sexo, idade, cor autodeclarada, profissional da saúde e uso do serviço de saúde), clínicas (hipertensão arterial sistêmica, diabetes melittus, neoplasias, doenças do aparelho respiratório, obesidade, doenças renais, doenças do aparelho cardiovascular), dos sinais e sintomas referentes à COVID-19 (febre, tosse, dor de garganta, dispnéia, diarreia, perda de paladar, vômito, cefaleia) e das características relacionadas à internação (realização de tomografia computadorizada, exames laboratoriais, leito de internação, leito pactuado pelo SUS, uso de ventilação mecânica e evolução do quadro).

Este estudo respeitou todos aspectos éticos em pesquisa, conforme a Resolução 466/2012 do Conselho Nacional de Saúde (CNS), conforme aprovação do CEP/UNEMAT, com CAAE: 28214720.9.0000.5166 e parecer de aprovação n. 3.903.714/2020. 


\section{RESULTADOS}

No período de junho a novembro de 2020 em um município polo da região Médio Norte de Mato Grosso, 93 pacientes estiveram hospitalizados devido a COVID-19, compondo assim, a mostra total desta pesquisa.

A população é predominantemente de indivíduos do sexo masculino (61,29\%), com idade maior ou igual a 60 anos $(59,14 \%)$, que se autodeclararam de cor parda $(52,69 \%)$. A maior parte dos afetados eram usuários do Sistema Único de Saúde (SUS) (65,59\%) e não trabalhavam na área da saúde (97,85\%) (Tabela 1).

Tabela 1 - Perfil sociodemográfico dos mato-grossenses internados por COVID-19 de junho a novembro de 2020.

\begin{tabular}{lcc}
\multicolumn{1}{c}{ VARIÁVEL } & n & \% \\
\hline Sexo & 36 & 38,71 \\
Feminino & 57 & 61,29 \\
Masculino & & \\
Idade & 20 & 21,51 \\
35 a 49 anos & 18 & 19,35 \\
50 a 59 anos & 55 & 59,14 \\
$\geq 60$ anos & & \\
Cor Autodeclarada & 34 & 36,56 \\
Branca & 49 & 52,69 \\
Parda & 4 & 4,30 \\
Negro & 6 & 6,45 \\
Outras & & 65,59 \\
Unidade de Saúde & 61 & 34,41 \\
SUS & 32 & \\
Particular & & \\
\hline
\end{tabular}

Fonte: Dos Autores.

Nos participantes desta pesquisa, 76,34\% dos que necessitaram de hospitalização relataram ser portadores de comorbidades pré-existentes. Dentre elas, as mais prevalentes foram a Hipertensão Arterial Sistêmica (HAS), Diabetes Melittus (DM) e doenças renais. De 
acordo com a estratificação sociodemográfica, os homens (46,24\%), idosos (48,39\%) e aqueles que se autodeclararam pardos $(39,78 \%)$, foram os que obtiveram maior prevalência de doenças pré-existentes (Tabela 2).

Tabela 2 - Perfil clínico de comorbidades da população estudada, baseado nas características sociodemográficas de indivíduos hospitalizados. Junho a novembro de 2020.

\begin{tabular}{|c|c|c|c|c|c|c|c|c|c|c|c|c|c|c|}
\hline \multirow[t]{2}{*}{ VARIÁVEL } & \multicolumn{2}{|c|}{ HAS } & \multicolumn{2}{|c|}{ DM } & \multicolumn{2}{|c|}{ Neoplasias } & \multicolumn{2}{|c|}{$\begin{array}{c}\text { Ap. } \\
\text { Respiratório }\end{array}$} & \multicolumn{2}{|c|}{ Obesidade } & \multicolumn{2}{|c|}{$\begin{array}{c}\text { Doenças } \\
\text { renais }\end{array}$} & \multicolumn{2}{|c|}{$\begin{array}{c}\text { Ap. } \\
\text { Cardiovascular }\end{array}$} \\
\hline & $\mathbf{n}$ & $\%$ & $\mathbf{n}$ & $\%$ & $\mathbf{n}$ & $\%$ & $\mathbf{n}$ & $\%$ & $\mathbf{n}$ & $\%$ & $\mathbf{n}$ & $\%$ & $\mathbf{n}$ & $\%$ \\
\hline \multicolumn{15}{|l|}{ Sexo } \\
\hline Feminino & 16 & 17,20 & 7 & 7,53 & 3 & 3,22 & 1 & 1,07 & 7 & 7,53 & 6 & 6,45 & 5 & 5,38 \\
\hline $\begin{array}{l}\text { Masculino } \\
\text { Idade }\end{array}$ & 22 & 23,66 & 15 & 16,13 & - & - & 2 & 2,15 & 7 & 7,53 & 8 & 8,60 & 2 & 2,15 \\
\hline 35 a 49 anos & 6 & 5,45 & 2 & 2,15 & - & - & - & - & 7 & 7,53 & 1 & 1,07 & - & - \\
\hline 50 a 59 anos & 7 & 7,53 & 4 & 4,30 & 1 & 1,07 & 1 & 1,07 & 2 & 2,15 & 3 & 3,22 & 1 & 1,07 \\
\hline$\geq 60$ anos & 25 & 26,88 & 16 & 17,20 & 2 & 2,15 & 2 & 2,15 & 5 & 5,38 & 10 & 10,75 & 6 & 6,45 \\
\hline \multicolumn{15}{|l|}{$\begin{array}{l}\text { Cor } \\
\text { Autodeclarada }\end{array}$} \\
\hline Branca & 14 & 14,58 & 7 & 7,53 & 2 & 2,15 & 2 & 2,15 & 6 & 6,45 & 3 & 3,22 & 4 & 4,30 \\
\hline Parda & 21 & 22,58 & 14 & 14,58 & 1 & 1,07 & 1 & 1,07 & 7 & 7,53 & 11 & 11,82 & 3 & 3,22 \\
\hline Negro & 1 & 1,07 & - & - & - & - & - & - & - & - & - & - & - & - \\
\hline Outras & 2 & 2,15 & 1 & 1,07 & - & - & - & - & 1 & 1,07 & - & - & - & - \\
\hline
\end{tabular}

Fonte: Dos Autores.

Os principais sintomas apresentados foram predominantemente dispneia/desconforto respiratório, tosse e febre. o tempo médio entre o início dos sinais e sintomas até a realização de exames diagnósticos foi de 7 dias.

Para diagnóstico da COVID-19 foram realizados exames laboratoriais e de imagem, sendo os exames que detectam antígeno para COVID-19/Teste rápido os que foram mais amplamente utilizados na classe laboratoriais com 63,44\% das amostras testadas. Entre os exames de imagem, apenas $36,56 \%$ das tomografias computadorizadas obtiveram imagens compatíveis com aspecto de vidro fosco, sugestivos clinicamente de COVID-19. Após o diagnóstico da doença, $66,67 \%$ das internações hospitalares foram predominantemente em leitos de enfermarias pactuadas pelo SUS (Tabela 3). 
Tabela 3 - Perfil de internações e de exames diagnósticos dos pacientes portadores de COVID-19 ( $n=93)$.

\begin{tabular}{|c|c|c|}
\hline Características de Internação & $\mathbf{n}$ & $\%$ \\
\hline \multicolumn{3}{|l|}{ Realização de Tomografia Computadorizada } \\
\hline Sugestiva para COVID-19 & 34 & 36,56 \\
\hline Não se aplica & 59 & 63,44 \\
\hline \multicolumn{3}{|l|}{ Exame Laboratoriais } \\
\hline Antígeno p/ COVID-19/Teste rápido & 59 & 63,44 \\
\hline RT-PCR & 31 & 33,33 \\
\hline Outros & 3 & 3,23 \\
\hline \multicolumn{3}{|l|}{ Leito de internação inicial } \\
\hline Enfermaria & 45 & 48,39 \\
\hline Isolamento & 12 & 12,90 \\
\hline Unidade de Terapia Intensiva & 36 & 38,71 \\
\hline \multicolumn{3}{|l|}{ Uso de ventilação mecânica inicial } \\
\hline Sim & 11 & 11,83 \\
\hline Não & 82 & 88,17 \\
\hline \multicolumn{3}{|l|}{ Evolução do Quadro } \\
\hline Alta/cura & 26 & 27,96 \\
\hline Transferência referenciada & 5 & 5,38 \\
\hline óbito & 54 & 58,06 \\
\hline Não informados & 8 & 8,60 \\
\hline
\end{tabular}

Fonte: Dos Autores.

Na tabela 3 verificou-se ainda que inicialmente 57 (61,29\%) dos pacientes foram internados em leitos de enfermaria e isolamento, sem uso de ventilação mecânica. Porém observou-se que destes, 36 (63,17\%) evoluíram para uso de ventilação mecânica em leitos de terapia semi-intensiva e terapia intensiva. A maioria dos participantes evoluiu para óbito (58,06\%) como prognóstico final, sendo 55,55\% dos óbitos entre o sexo masculino. A média de dias entre a internação e o prognóstico final foi de 12 dias.

\section{DISCUSSÃO}


O perfil dos pacientes hospitalizados pela COVID-19 foi composto predominantemente por indivíduos do sexo masculino, idosos e que se autodeclararam pardos. Em estudos nacionais realizados com a população geral é possível encontrar variações no perfil sociodemográfico em relação à incidência de COVID-19 no Brasil. Um exemplo está na pesquisa realizada por Lima et al. (2020) no Ceará (BR), cuja população de pacientes infectados foi representada por adultos jovens, com faixa etária de 20 a 39 anos e sexo feminino. Outro perfil semelhantemente foi encontrado por Ferreira et al. (2020) no Espírito Santo (BR) e no Rio Grande do Norte (BR) por Galvão; Roncalli (2020), onde além de serem infectadas mulheres e adultos jovens, a população foi representada por aqueles que se autodeclararam pardos. Vale ressaltar que tais estudos foram realizados com dados secundários gerais da população acometida pela COVID-19 baseados nas informações dos Sistemas de Informação de Saúde.

Estudo realizado por Jailon; Berthenet; Garlanda (2019) sobre a influência do sexo na resposta do sistema imune inata, consideraram que a maior incidência de infecções ocorre em mulheres, e que pode relacionar-se com o importante papel que os cromossomos $\mathrm{X}$ do sexo feminino e os hormônios sexuais desempenham no sistema imunológico das mulheres. Apesar do número de casos ser maior entre o sexo feminino, alguns estudos identificaram que o maior índice de letalidade da COVID-19 se dá entre os homens (GALVÃO; RONCALLI, 2020; LIMA et al., 2021).

Barbosa et al. (2020) e Escobar; Rodrigues; Monteiro (2021), obtiveram como resultado uma correlação entre a taxa de incidência da COVID-19 e a cor da pele. Bernardes; Arruzzo; Monteiro (2020) afirmam que a composição racial dos vulneráveis da COVID-19 no Brasil, envolvendo aspectos socioeconômicos e de saúde, é predominantemente autodeclarada pardas ou negras, o que favorece a uma maior incidência da doença nesta população visto que as vulnerabilidades sociais e econômicas nas quais vivenciam favoreçam situações de risco para a contaminação pela COVID-19, podendo ser um fator explicativo dos achados do presente estudo, com predominância de pessoas pardas.

Apesar dos estudos nacionais apontarem que a população jovem é a mais acometida pela COVID-19 (FERREIRA et al., 2020; GALVÃO; RONCALLI, 2020; LIMA et al., 2020) neste estudo a população idosa foi a que representou o maior quantitativo. Galvão; Roncalli (2020) verificaram que ser idoso representava de 2,87 a 7,06 de chances a mais de desenvolver casos 
graves da doença e consequentemente aumento na mortalidade. No estudo nacional realizado por Niquini et al. (2020) com base nos dados disponibilizados pelo Ministério da Saúde sobre os casos de hospitalização no Brasil por SRAG-COVID, $45,2 \%$ dos indivíduos hospitalizados por COVID-19 eram idosos. Este alto índice pode ser explicado porque nesta faixa de idade há maior prevalência de comorbidades, o que determina maiores complicações com apresentações de formas mais severas da COVID-19, com elevação da letalidade nesse segmento populacional (ESCOBAR; RODRIGUEZ; MONTEIRO, 2021).

A presença de comorbidades em todo país se tornou com o tempo um problema de saúde pública ao considerar a grande prevalência na população. A presença de doenças crônicas ocasiona múltiplas alterações fisiopatológicas, alterando a resposta imunológica e os mecanismos de adaptação do organismo quando somadas a episódios agudos desta comorbidades bem como de outras afecções que a que seus portadores possam ser acometidos. A combinação de diferentes problemas crônicos de saúde somados as vulnerabilidades de saúde, favorecem o desenvolvimento de novos processos inflamatórios, aumentando a suscetibilidade da contrair doenças infecciosas agudas como a COVID-19 e outros agravos (NUNES et al., 2020; SHEPPARD et al., 2020). Dentre os pacientes hospitalizados, a maioria era portador de comorbidades pré-existentes, sendo hipertensão arterial, diabetes e doenças renais as mais prevalentes. Na Turquia, Durmus; Guneysu (2020) observaram que mais da metade da população infectada possuía hipertensão arterial, seguida de doenças cardiovasculares e diabetes. Liu et al. (2020) e Suleyman; Fadel; Malette (2020) puderam verificar em Wuhan na China e em Detroit nos Estados Unidos, respectivamente, que além destas, as doenças renais pré-existentes também eram prevalentes em sua população de pacientes hospitalizados em decorrência da infecção pelo SARS-CoV-2.

Ao analisar a fisiopatologia destas comorbidades em pacientes com COVID-19, observa-se que há uma correlação entre os mecanismos fisiológicos e a ação do vírus no organismo. Um destes mecanismos está no sistema Renina-Angiotensina-Aldosterona, onde a Enzima Conversora de Angiotensina 2 é responsável pela regulação cardiovascular da pressão sanguínea na função de vasoconstrição, que em níveis elevados ocasiona uma desregulação da função vascular, instalando assim um quadro de hipertensão arterial. A expressão aumentada da ECA-2 em pacientes hipertensos ocorre devido a necessidade do controle dos níveis pressóricos por meio de medicamentos, como inibidores da enzima de conversão da angiotensina ou bloqueadores do receptor da angiotensina II, o que facilita a 
entrada da SARS-CoV-2 nas células, visto que o receptor celular para o vírus da COVID-19 possui afinidade pelos receptores da ECA-2 o que proporciona sua entrada e desenvolvimento no organismo daqueles cuja sua expressão esteja descompensada (PARK et al., 2020; SHEPPARD et al., 2020; WANG et al., 2021; ZHANG et al., 2020).

Dentre as principais comorbidades prevalentes neste estudo, o diabetes mellitus é uma doença crônica que ocorre devido a insuficiência ou ausência da produção do hormônio insulina no organismo e que ocasiona um quadro de hiperglicemia (OMS, 2016). Devido a descompensação glicêmica o diabetes ocasiona danos teciduais, o que facilita o desenvolvimento de condições incapacitantes e diversas complicações à saúde, principalmente em indivíduos que são acometidos pela COVID-19, pois o diabetes possui características semelhantes a doenças infecciosas, quando não controlada é capaz de ocasionar liberação de mediadores inflamatórios resultantes de uma disfunção endotelial, neuropática e também do enfraquecimento da resposta imune inata do organismo, que quando combinadas as alterações metabólicas ocasionadas pela COVID-19 podem ser fatores predisponentes ao agravamento do quadro clínico (ALVES et al., 2020; LIPPI; SANCHISGOMAR; HENRY, 2020).

Além da infecção das vias aéreas e da síndrome do desconforto respiratório aguda, a insuficiência renal é comum em pacientes hospitalizados com COVID-19. Classificadas em insuficiência aguda (IRA) e crônica (IRC), são consideradas fatores de risco para agravamento do quadro clínico em pacientes acometidos com COVID-19, devido ao aumento da creatinina sérica e da redução da taxa de filtração glomerular ocasionado a redução da atividade renal pelas lesões teciduais e alterações fisiológicas (CAMPOS et al., 2020; CHUEH et al., 2020; YANG et al., 2020). Quando a insuficiência renal se torna crônica há uma lesão progressiva e irreversível de unidades funcionais do rim, o que faz o paciente portador de IRC necessitar de tratamentos imunossupressores específicos para controle da doença e em sua maioria realizar contínuas sessões de hemodiálise, que os tornam propensos a frequentar e até mesmo permanecer em instituições médicas/hospitalares para cuidados de médio a longo prazo, bem como expostos a uma maior vulnerabilidade de adquirir COVID-19 e desenvolver a forma complicada da COVID-19 (DINO; CAMPOS, 2017; CHO et al., 2021; TAJI et al., 2021). A COVID-19 pode manifestar-se desde a forma assintomática até formas graves com importante comprometimento do sistema respiratório (DOREMALEN et al., 2020). Os sinais e sintomas da 
doença são compostos principalmente por febre, tosse seca e dispneia com possibilidade de complicações, principalmente pneumonia, síndrome respiratória aguda grave (SRAG) e óbito (CHEN et al., 2020; NUNES et al., 2020).

Neste estudo, os sinais e sintomas mais prevalentes na população hospitalizada foram predominantemente dispneia, tosse e febre. Este perfil de sintomas ocasionados pela COVID19 tem sido padrão em diversos estudos, como no Espírito Santo (BR), nos Estados Unidos, na China e Coreia do Sul (CHEN et al,, 2020; PARK et al., 2020; SULEYMAN; FADEL; MALETTE 2020;). Apesar da infecção ocasionar afecções principalmente de caráter respiratório, outros sintomas são comumente percebidos como dor de garganta, fraqueza, dores musculares e sintomas gastrointestinais, como náusea, vomito e episódios de diarreia (SOUZA; LEAL; SANTOS, 2020). 0 tempo médio de aparecimento dos primeiros sinais e sintomas da COVID19 na população deste estudo foi de sete dias. Nas capitais brasileiras, Lima et al. (2021) observaram que esse tempo médio foi de 10,2 dias. Este intervalo compreende o período de incubação viral, que pode variar de 1 a 14 dias para o aparecimento dos primeiros sinais da doença (DOREMALEN et al., 2020).

O desafio de diferenciá-la de outras síndromes gripais está presente no cotidiano dos serviços assistenciais de saúde, e deve as manifestações clínicas características de outras afecções febris e agudas, por esse motivo o diagnóstico específico tornou-se o principal mecanismo de confirmação para novos casos de COVID-19. Através de investigações clínicasepidemiológicas e realização de exames laboratoriais e de imagem, como testes rápidos, PCR e Tomografia Computadorizada (TC) por exemplo, a identificação de indivíduos infectados favoreceu em muitos casos o tratamento adequado, bem como a instalação de medidas de distanciamento social, com a quebra da cadeia de transmissão da doença (BRASIL, 2020b; LIMA et al., 2021).

A maioria dos pacientes internados em Mato Grosso foram diagnosticados por meio de exames Antígeno para COVID-19 - Teste rápido. Lima et al. (2021) observaram que a realização de testes-rápidos foi a mais prevalente em todas regiões do Brasil entre março e agosto de 2020, sendo as regiões Nordeste e Sudeste as que mais utilizaram este tipo de diagnóstico (BRASIL, 2020c, 2020; ZHAO et al., 2020). Esta grande prevalência pode ser justificada pelo aumento na facilidade de acesso aos testes-rápidos, pois em muitas regiões este tipo de teste se tornou uma opção para a população geral, pois não necessitam de 
hospitalização para serem realizados e oferecem resultado em curto espaço de tempo. Da mesma forma esta alta adesão a este tipo de testagem pode justificar-se pelos esforços do Ministério da Saúde para atender à recomendação da OMS, ampliando a oferta de testes para diagnosticar a COVID-19, com menor tempo entre o início dos sinais e sintomas e o diagnóstico final, outro fator é que tais testes exigem menores custos e tempo de execução, mão de obra e tecnologias para sua realização comparado a outros testes como o RT-PCR e exames de imagem como Tomografia Computadorizada (BRASIL, 2020d; TOLIA; CHAN; CASTILLO, 2020).

Além dos testes laboratoriais, os exames de imagem têm sido fundamentais no complemento ao diagnóstico para COVID-19 e do direcionamento da estratégia de assistência clínica, sendo a TC o exame mais realizado. A TC favorece a verificação de lesões e alterações físicas características da infecção pelo novo coronavírus. Devido a doença afetar preferencialmente os pulmões, as manifestações e características da TC mais comumente vistas são achados na pneumonia, em que há o comprometimento bilateral pulmonar com apresentação de opacidade em vidro fosco com distribuição subpleural (ZHOU et al., 2021). Entre os pacientes internados por COVID-19 pertencentes a este estudo, apenas 36,56\% realizaram este exame, porém valores maiores puderam ser observados em estudos como o de Guan et al. (2020) e Bastos et al. (2020) onde 56,4\% e 91,86\% dos pacientes, respectivamente, realizaram TC e obtiveram achados de opacidade em vidro fosco característico para COVID19, o que pode remeter ao acesso facilitado as tecnologias em grandes centros médicos, diferentemente da realidade mato-grossense.

Nesta pesquisa, as internações hospitalares foram predominantemente em leitos pactuados pelo SUS, porém com a evolução da doença 63,17\% dos pacientes evoluíram para internações em leitos de UTI e unidades semi-intensivas, pois necessitaram de cuidados intensivos associados a medidas de suporte à vida como uso de ventilação mecânica (VM). Em estudo nacional realizado com base nos dados do Sistema de Informação Hospitalar (SIH) no período de fevereiro a junho de 2020, 22,6\% das hospitalizações no país necessitaram de leitos de UTI (ANDRADE et al., 2020). Em Porto Alegre (SC) 32,9\% dos pacientes hospitalizados foram admitidos à UTI e 20,5\% necessitaram de VM invasiva, enquanto na Suécia apenas $10 \%$ dos pacientes necessitaram ser internados em UTI requerendo uso de VM (BASTOS et al., 2020; SVENSSON et al., 2021). 
De acordo com o SIH, no ano de 2020 do valor total pago por todas as internações do COVID-19 pelo SUS, 66,4\% do orçamento foi destinado para leitos de UTI em todo país, investimento direcionado a ampliação e preparo de novos leitos para atender a demanda crescente da doença (ANDRADE et al., 2020; CASTRO et al., 2019). Rache et al. (2020) observaram que de acordo com as regiões de saúde, a quantidade de leitos de UTI pactuados pelo SUS e equipamentos de VM eram insuficientes, corroborando com este achado, Moreira (2020) ao analisar dados dos estabelecimentos de saúde, concluiu que o Brasil contava com 29.891 unidades de UTI em janeiro de 2020, sendo que apenas 47,15\% destas eram pertencentes e financiadas pelo SUS.

Rache et al. (2020) obtiveram como resultado que a disparidade regional na distribuição de leitos de UTI do SUS e equipamentos de VM estão diretamente relacionados à região do país, bem como seus fatores culturais e sociodemográfico, haja visto que a maior concentração de leitos e suporte para cuidados intensivos estarem na região Sudeste, e em escala decrescente no Nordeste, Sul, Centro-Oeste e Norte. Esse fato aliado ao número exponencial de infecções decorrentes da pandemia de COVID-19 em 2020, tende a sobrecarregar, em especial os Estados e regiões mais vulneráveis afetando significativamente a população brasileira, ao gerar uma enorme sobrecarga monetária ao SUS, ocasionada devido a existência de um sistema de saúde afetado pela desigualdade, já que a distribuição de recursos em saúde não é igualitária entre as unidades federativas do Brasil (MENDONÇA et al., 2020). Todavia, o subfinanciamento e a gestão inadequada do SUS minaram sua estrutura em plena pandemia. Apesar do aumento da oferta de leitos hospitalares de cuidados gerais e intensivos e da construção de hospitais de campanha, tem sido cada vez mais essencial a identificação de regiões mais vulneráveis, a fim de otimizar o uso de serviços existentes, dimensionando recursos que são necessários para fortalecer a capacidade de resposta do SUS em âmbito regional e local (ANDRADE et al., 2020; RACHE et al., 2020).

Devido ao alto número de hospitalizações, a ocorrência de casos graves da COVID-19 somados a fragilidade da infraestrutura dos serviços de saúde, mais da metade dos pacientes participantes deste estudo evoluíram para óbito, sendo mais prevalente entre os homens. Esse comportamento epidemiológico foi semelhante em Rondônia, onde 63,1\% dos óbitos por COVID-19 foram entre indivíduos do sexo masculino em todas faixas de idade. No sudeste dos EUA $40,4 \%$ dos pacientes que desenvolveram casos graves da doença tiveram óbito como 
desfecho final, e ser do sexo masculino foi significativamente associado à mortalidade por COVID-19 (ESCOBAR; RODRIGUEZ; MONTEIRO, 2021; SULEYMAN; FADEL; MALETTE, 2020).

Ser do sexo masculino também foi um fator associado a um maior risco de morrer em decorrência da COVID-19, pois homens apresentam probabilidade de sobrevivência acumulada menor que mulheres, bem como um risco de óbito $45 \%$ maior que o das mulheres (GALVÃo; RONCALLI, 2020). Sabe-se que historicamente indivíduos do sexo masculino são os que menos acessam os serviços de prevenção e promoção de saúde, da mesma forma a maior probabilidade de ocorrência de óbito em homens pode ser explicada por estes procurarem tardiamente os serviços de saúde em casos mais graves da doença (CAVALCANTE; ABREU, 2020; TEIXEIRA, 2016).

Diante da emergência e gravidade da COVID-19 para a sociedade mundial, estudos como estes são de extrema relevância para a área da saúde, visto que ao analisar as comorbidades prevalentes em pacientes hospitalizados pela COVID-19, intervenções de saúde podem ser realizadas de formas eficazes visando a diminuição das chances de complicações relacionada a presença de doenças pré-existentes. 0 conhecimento das comorbidades mais prevalentes na população estudada, permite também o conhecimento do perfil de saúde e das vulnerabilidades vivenciadas pela população estudada, bem como intervir efetivamente na promoção de saúde e prevenção de agravos relacionados à incidência da COVID-19 concomitante às condições crônicas de saúde. As limitações de um estudo ecológico não se restringem somente as dificuldades de construi-lo, mas relacionam-se principalmente à fonte de onde os dados são coletados. As limitações de utilização desse material encontramse na subnotificação de informações relacionadas a infecção pela COVID-19. Dados sociodemográficos, de saúde e informações relacionadas às hospitalizações na pandemia têm sido falhamente registrados.

\section{CONSIDERAÇÕES FINAIS}

A pandemia da COVID-19 trouxe consigo a emergência de uma doença infecciosa que afeta especificamente o aparelho respiratório, que varia de quadros leves a graves, 
principalmente em indivíduos portadores de comorbidades pré-existentes. O perfil dos pacientes hospitalizados pela COVID-19 participantes desta pesquisa foi composto predominantemente por indivíduos do sexo masculino, idosos e que se autodeclararam pardos. Verificou-se que o fator idade e sexo influenciam na incidência de casos novos da doença, e que possuir comorbidades é fator de risco para desenvolvimento de complicações. Destacou-se que as doenças crônicas mais prevalentes foram a hipertensão arterial, diabetes melittus e doenças renais, comorbidades estas que alteram a fisiologia e homeostasia do organismo favorecendo a infecção pela COVID-19. O diagnóstico foi predominantemente realizado por meio de Testes-Rápidos em busca de detecção de antígenos, o que ressaltou o acesso facilitado a esses mecanismos para identificação de indivíduos infectados. A maior parte das internações foram em leitos pactuados pelo SUS, principalmente em leito de UTI. Apesar das intervenções terapêuticas realizadas, mais da metade da população portadora de comorbidades evoluiu para óbito, o que reflete a gravidade da doença em relação aos fatores de riscos que as comorbidades representam para os pacientes hospitalizados.

Estudos como estes permitem o conhecimento da realidade de saúde enfrentada, bem como o comportamento da COVID-19 em pacientes hospitalizados devido a doença, seu perfil clínico-epidemiológico e sociodemográfico. Ao se conhecer a população afetada e seus fatores condicionantes de saúde, ações estratégias governamentais poderão ser realizadas de formas eficazes ao garantir total acesso aos meios de recuperação dos agravos ocasionados pela COVID-19. Além disso, observa-se que a produção científica que analise as hospitalizações em regiões do Centro-Oeste, são ainda pouco incipientes, o que torna esta pesquisa fundamental para a visualização e compreensão da pandemia da COVID-19 em seu contexto de saúde pública na região.

\section{REFERÊNCIAS}

ALVES, D.A.N.S.; NASCIMENTO, G.I.L.A.; CASTANHA, E.R.; LUNA, J.E.L.; SOBRAL, E.F.M.; BRANDÃO, W.A.; MOREIRA, K.A.; MENDES, J.S.; FILHO, M.C.; BARROS, D.M.; FALCÃO. R. E. A. Prevalência de comorbidades na Síndrome Respiratória Aguda Grave em pacientes acometidos por COVID-19 e outros agentes infecciosos. Research, Society And Development, [S.L.], v. 9, n. 11, p. 1-18, 1 dez. 2020. Research, Society and Development. Disponível em: http://dx.doi.org/10.33448/rsd-v9i11.10286. Acesso em 24 fev. 2021. 
AMMAR, A.; CHTOUROU, H.; BOUKHRIS, O.; TRABELSI, K. et al. O COVID-19 Confinamento Doméstico Impacta Negativamente a Participação Social e a Satisfação com a Vida: Um Estudo Multicêntrico Mundial. Int J Environ Res Saúde Pública, v. 17, n. 17, p. 6237, Dec. 2020. Disponível em: https://www.ncbi.nlm.nih.gov/pmc/articles/PMC7503681/. Acesso em 26 jan. 2021.

ANDRADE, C. L. T.; PEREIRA, C.C.A.; MARTINS, M.; LIMA, S.M.L.; PORTELA, MC.C. COVID-19 hospitalizations in Brazil's Unified Health System (SUS). Plos One, [S.L.], v. 15, n. 12, p. 1-17, 10 dez. 2020. Public Library of Science (PLoS). Disponível em: http://dx.doi.org/10.1371/journal.pone.0243126 . Acesso em 01 fev. 2021.

ARAUJO, L. S.; SHIOMATSU, G.Y.; NINOMIYA, V.Y.; CARVALHO, R.T. Por que os diabéticos fazem parte do grupo de risco da covid-19. Disponível em: https://coronavirus.saude.mg.gov.br/blog/93-covid-19-empacientes-diabeticos . Acesso em 27 jan. 2021.

ASKIN, L.; TANRIVERDI, O.; ASKIN, H. S. O Efeito da Doença de Coronavírus 2019 nas Doenças Cardiovasculares. Arquivos Brasileiros de Cardiologia, [S.L.], v. 114, n. 5, p. 817-822, maio 2020. Sociedade Brasileira de Cardiologia. Disponível em: http://dx.doi.org/10.36660/abc.20200273 . Acesso em 15 fev. 2021.

BARBOSA, I. R.; GALVÃO, M.H.R.; SOUZA, T.A.; GOMES, S.M.; MEDEIROS, A.A.; LIMA, K.C. Incidence of and mortality from COVID-19 in the older Brazilian population and its relationship with contextual indicators: an ecological study. Revista Brasileira de Geriatria e Gerontologia, [S.L.], v. 23, n. 1, p. 1-11, 2020. FapUNIFESP (SciELO). Disponível em: http://dx.doi.org/10.1590/1981-22562020023.200171. Acesso em 05 fev. 2021.

BASTOS, G.A.N.; AZAMBUJA, A.A.; POLANCZYK, C.A.; GRÄF, D.D.; ZORZO, I.W.; MACCARI, J.G.;HAYGERT, L.S.; NASI, L.A.; GAZZANA, M.B.; BESSEL, M.; PITREZ, P.M.; OLIVEIRA, R.P.; SCOTTA, M.C. Clinical characteristics and predictors of mechanical ventilation in patients with COVID-19 hospitalized in Southern Brazil. Rev Bras Ter Intensiva. v. 32, n. 4, p. 487-492, 2020. Disponível em: http://dx.doi.org/10.5935/0103-507X.20200082. Acesso em 24 fev. 2021.

BERNARDES, J. A.; ARRUZZO, R. C.; MONTEIRO, D. M. L. V.. Geografia e COVID-19: neoliberalismo, vulnerabilidades e luta pela vida. Revista Tamoios, [S.L.], v. 16, n. 1, p. 188-205, 7 maio 2020. Universidade de Estado do Rio de Janeiro. Disponível em: http://dx.doi.org/10.12957/tamoios.2020.50645 . Acesso em 16 fev. 2021.

BOTERO, D.M.R.; OMAR, AM.S.; SUN, H.K.; MANTRI, N.; FORTUZI, K.; CHOI, Y.; ADRISH, M.; NICU, M.; BELLA, J.N.; CHILIMUIR, S. COVID-19 na população de pacientes saudáveis: caracterização demográfica e fenotípica clínica e preditores de resultados hospitalares. Arterioscler Thromb Vasc Biol, v.40, n.11, p. 2764-2775, 2020. DOI http://dx.doi.org/10.1161/ATVBAHA.120.314845. Acesso em 05 jan. 2021.

BRASIL, 2020a. Ministério da Saúde. Painel Coronavírus. Disponível em:<https://covid.saude.gov.br/>. Acesso em 09 jan. 2021.

BRASIL. Ministério da Saúde. Ministério da Saúde. Orientações para manejo de pacientes com CoVID-19. Ministério da Saúde, Brasília, p. 1-49, 2020b. Disponível em: https://portalarquivos2.saude.gov.br/images/pdf/2020/marco/24/20200323-ProtocoloManejo-ver05.pdf

BRASIL, Ministério da Saúde (BR). Secretaria de Vigilância em Saúde. Guia de Vigilância Epidemiológica. Emergência de saúde pública de importância nacional pela doença pelo coronavírus 2019: vigilância de síndromes respiratórias agudas COVID-19. [Internet]. Brasília: Ministério da Saúde; 2020c [citado 2020 ago 29]. 57 p. Disponível em: https://covid19-evidence.paho.org/handle/20.500.12663/2114.

BRASIL, Ministério da Saúde (BR). Saúde amplia testes para profissionais de saúde e segurança [Internet]. Brasília: Ministério da Saúde; 2020d [citado 2020 ago 30]. Disponível em: https://www.saude.gov.br/noticias/agencia-saude/46596-saude-amplia-testes-paraprofissionais-de-saude-eseguranca. Acesso em 01 mar. 2021.)

CALÓ, R.S.; ASSIS, J.M.V.; GUENKKA, T.M.; PIRES, J.C.S.; ANDRADE, A.C.S.; SOUZA, R.A.G. Perfil epidemiológico dos óbitos por Coronavírus (COVID -19) em Mato Grosso. Disponível em: https://doi.org/10.36489/saudecoletiva.2020v10i56p3044-3055. Acesso em 24 jan. 2021. 
CAMPOS, M. R.; SCHRAMM, J.M.A.; EMMERICK, I.C.M.; RODRIGUES, J.M.; AVELAR, F.G.; PIMENTEL, T.G. Carga de doença da COVID-19 e de suas complicações agudas e crônicas: reflexões sobre a mensuração (daly) e perspectivas no sistema único de saúde. Cadernos de Saúde Pública, [S.L.], v. 36, n. 11, p. 1-14, 2020. FapUNIFESP (SciELO). Disponível em: http://dx.doi.org/10.1590/0102-311x00148920 . Acesso em 04 fev. 2021.

CARRATURO, F.; GIUDISSE, C.D.; MORELLI, M.; CERULLO, V.; LIBRALATO, G.; GALDIERO, E.; GUIDA, M. Persistência de SARS-CoV-2 no meio ambiente e risco de transmissão de COVID-19 de matrizes e superfícies ambientais. Environ Pollut, v. 265, Dec. 2020. Disponível em: https://www.ncbi.nlm.nih.gov/pmc/articles/PMC7280109/ . Acesso em 26 jan. 2021.

CASTRO, M. C.; MASSUDA, A.; ALMEIDA, G.; MENEZES-FILHO, N.A.; ANDRADE, M.V.; NORONHA, K.V.M.S.; ROCHA, R.; MACINKO, J.; HONE, T.; GIOBANELLA, L.; MALIK, A.M.M; WERNECK, H.; FACHINI, L.A.; ATUN, R. Brazil's unified health system: the first 30 years and prospects for the future. Lancet, v. 394, n. 10195, p. 345- 356, 2019. Disponível em: https://doi.org/10.1016/S0140-6736(19)31243-7. Acesso em 27 fev. 2021.

CAVALCANTE, J. R.; ABREU, A. J. L. COVID-19 no município do Rio de Janeiro: análise espacial da ocorrência dos primeiros casos e óbitos confirmados. Epidemiol. Serv. Saúde, Brasília , v. 29, n. 3, e2020204, 2020 Disponível em: http://dx.doi.org/10.5123/s1679-49742020000300007. Acesso em 13 fev. 2021.

CHEN, N.; ZHOU, M.; QU, J.; GONG, F.; HAN, Y.; QIU, Y.; WANG, J.; LIU, Y.; WEI, Y.; XIA, J.; YU, T.; ZHANG, X.; ZHANG, L. Epidemiological and clinical characteristics of 99 cases of 2019 novel coronavirus pneumonia in wuhan, china: a descriptive study. The Lancet, v. 395, n. 10223, p. 1690-1691, 2020. Disponível em: http://dx.doi.org/10.1016/S0140-6736(20)30211-7. Acesso em 04 fev. 2021.

CHO, S.; SUNG-SOO, P.; MIN-KYU, C.; BAE, J.Y.; LEE, D.; KI, D. Prognosis Score System to Predict Survival for COVID-19 Cases: a Korean Nationwide Cohort Study. J Med Internet Res, v.23, n. 2, e26257, 2021. Disponível em: http://dx.doi.org/10.2196/6257 . Acesso em 17 fev. 2021.

CHUEH, T.; ZHENG, C.; HOU, Y.; LU, K.. Novel Evidence of Acute Kidney Injury in COVID-19. Journal of Clinical Medicine, [S.L.], v. 9, n. 11, p. 1679-1684, 3, 2020. MDPI AG. Disponível em: http://dx.doi.org/10.3390/jcm9113547 . Acesso em 22 fev. 2021.

COSTA, J. A.; SILVEIRA, J.A.; SANTOS, S.C.M.; NOGUEIRA, P.P. Implicações Cardiovasculares em Pacientes Infectados com Covid-19 e a Importância do Isolamento Social para Reduzir a Disseminação da Doença. SBC, Rio de Janeiro, Dec. 2020. Disponível em: https://www.scielo.br/pdf/abc/v114n5/0066-782X-abc-114-05-0834.pdf .Acesso em 28 jan. 2021.

CUSCHIERI. S.; GRECH, S. População com obesidade em risco de complicações COVID-19. Glob Health Epidemiol Genom, v.5, n. 6, 2020. DOI 10.1017 / gheg.2020.6. Disponível em: https://www.ncbi.nlm.nih.gov/pmc/articles/PMC7681109/ . Acesso em 05 jan. 2021.

DAVID, H. S. L.; ACIOLI, S.; SILVA, M.R.F.; BONETTI, O.P.; PASSO, H. Pandemia, conjunturas de crise e prática profissional: qual o papel da enfermagem diante da Covid-19?. Rev. Gaúcha Enferm, Porto Alegre, v. 42, 2020. DOI https://doi.org/10.1590/1983-1447.2021.20190254. Disponível em: https://www.scielo.br/scielo.php?pid=S1983-14472021000200702\&script=sci_arttext\&tlng=pt. Acesso em 05 jan. 2021.

DINO, B. D.; CAMPOS, R.. Insuficiência renal crônica e suas implicações para os sistemas metabólicos. Revista UNIANDRADE. Disponível em: http://dx.doi.org/10.18024/1519-5694/revuniandrade.v18n3p149-156 . Acesso em 27 fev. 2021.

DOREMALEN, N.V.; MORRIS, D.H.; HOLBROOK, M.G.; GAMBLE, A.; WILLIAMSON, B.N.; TAMIN, A.; LLOYD-SMITH, J.O.; WIT, E. Aerosol and Surface Stability of SARS-CoV-2 as Compared with SARS-CoV-1. The New England Journal of Medicine. Massachusetts, 2020. Disponível em: https://doi.org/10.1056/NEJMc2004973. Acesso em: 14 fev. 2021. 
DURMUS, E.; GUNEYSU, F.. Evaluation of re-hospitalized COVID-19 patients in a hospital. Revista da Associação Médica Brasileira, [S.L.], v. 66, n. 12, p. 1690-1695, dez. 2020. FapUNIFESP (SciELO). Disponível em: http://dx.doi.org/10.1590/1806-9282.66.12.1690. Acesso em 16 fev. 2021.

EJAZ, H.; ALSRHANI, A.; ZAFAR, A.; JAVED, H.; JUNAID, K.; ABDALA, A.E.; ABOSALIF, K.O.A.; AHMERD, Z.; YOUNAS, S. COVID-19 e comorbidades: impacto deletério em pacientes infectados. J Infect Public Health, v. 13, n. 12, p. 1833-1839, 2020. Disponível em: https://www.ncbi.nlm.nih.gov/pmc/articles/PMC7402107/. Acesso em: 01 fev. 2021.

ESCOBAR, A. L.; RODRIGUEZ, T. D. M.; MONTEIRO, J. C. Letalidade e características dos óbitos por COVID-19 em Rondônia: estudo observacional. Epidemiologia e Serviços de Saúde, [S.L.], v. 30, n. 1, p. 1-10, 2021. FapUNIFESP (SciELO). Disponível em: http://dx.doi.org/10.1590/s1679-49742021000100019. Aceso em 27 fev. 2021.

FERREIRA, A. D. S.; PEROVANO, L.S.; BARVOZA, L.I.; NASCIMENTO, W.M.; SILVA, F.M.; REIS, E.C. Perfil sociodemográfico dos pacientes confirmados para Covid-19 residentes no Espírito Santo, Brasil. Atoz: novas práticas em informação e conhecimento, [S.L.], v. 9, n. 2, p. 216-223, 21 dez. 2020. Universidade Federal do Parana. Disponível em: http://dx.doi.org/10.5380/atoz.v9i2.76179 . Acesso em 14 fev. 2021.

FUNDAÇÃO OSWALDO CRUZ - Fiocruz. Covid nas favelas. Disponível em: https://portal.fiocruz.br/observatorio-covid-19-covid-nas-favelas . Acesso em 07 jan. 2021.

FUNDAÇÃO OSWALDO CRUZ - Fiocruz. Impactos sociais, econômicos, culturais e políticos da pandemia. Disponível em: https://portal.fiocruz.br/impactos-sociais-economicos-culturais-e-politicos-da-pandemia . Acesso em 07 jan. 2021.

GROSSO, S. Painel COVID-19: Secretaria de estado de saúde de mato grosso. Disponível em: http://www.saude.mt.gov.br/painelcovidmt2/ . Acesso em 09 jan. 2021.

GUAN, W.; LIANG, W.; ZHAO, Y.; LIANG, H.; et al. Comorbidade e seu impacto em 1.590 pacientes com COVID-19 na China: uma análise nacional. Eur Respir J, v. 55, n.5, 2020. Disponível em: https://www.ncbi.nlm.nih.gov/pmc/articles/PMC7098485/ . Acesso em 27 jan. 2021.

GALVÃO, M. H. R.; RONCALLI, A. G. Fatores associados a maior risco de ocorrência de óbito por COVID-19: análise de sobrevivência com base em casos confirmados. Revista Brasileira de Epidemiologia, [S.L.], v. 23, p. 1-10, 2020. FapUNIFESP (SciELO). Diponível em: http://dx.doi.org/10.1590/1980-549720200106. Acesso em 28 fev. 2021.

HU, L.; CHEN, H.; FU, Y.; GAO, Z.; LONG, H.; WANG, J.; REN, H,. ZUO, Y.; LI, H.; WNAG, J.; YU, W.; LUI, J.; SHAO, C.; HAO, J.; WANG, C.; MA, Y.; WANG, Z.; YANAGIHARA, R.; DENG, Y. Fatores de risco associados a resultados clínicos em pacientes hospitalizados com 323 COVID-19 em Wuhan, China. Clin Infect Dis, 2020. Disponível em: https://www.ncbi.nlm.nih.gov/pmc/articles/PMC7197620/ . Acesso em 27 jan. 2021.

JAILLON, S.; BERTHENET, K.; GARLANDA, C. Sexual dimorphism in innate immunity. Clin Rev Allergy Immunol, v. 56, n. 3, p. 308-321, 2019. Disponível em: https://pubmed.ncbi.nlm.nih.gov/28963611/ . Acesso em 22 fev. 2021.

LIMA, D. L. F.; DIAS, A.A.; RABELO, R.S.; CRUZ, I.D.; COSTA, S.C.; NIGRI, F.M.N.; NERI, J.R. COVID-19 no estado do Ceará, Brasil: comportamentos e crenças na chegada da pandemia. Ciência \& Saúde Coletiva, [S.L.], v. 25, n. 5, p. 1575-1586, maio 2020. FapUNIFESP (SciELO). Disponível em: http://dx.doi.org/10.1590/141381232020255.07192020. Acesso em 18 fev. 2021.

LIMA, F.E.T.; ALBURQUERQUE, N.L.S.; FLORENCIO, S.S.G.; FONTENELE, M.G.M.; QUEIROZ, A.P.O.; LIMA, G.A.; FIGUEIREDO, L.M.; AMORIM, S.M.C.; BARBOSA, L.P. Intervalo de tempo decorrido entre o início dos sintomas e a realização do exame para COVID-19 nas capitais brasileiras, agosto de 2021. Epidemiologia Serviço de Saúde, [S.L.], p. 1-10, 4 nov. 2020. FapUNIFESP (SciELO). Disponível em: http://dx.doi.org/10.1590/s167949742021000100021 . Acesso em 27 fev. 2021. 
LIPPI, G.; MATTIUZZI, C.; SANCHIS-GOMAR, F.; HENRY, B. M. Clinical and demographic characteristics of patients dying from COVID-19 in Italy vs China. Journal of Medical Virology, [S.L.], v. 92, n. 10, p. 1759-1760, 2 jun. 2020. Wiley. Disponível em: http://dx.doi.org/10.1002/jmv.25860 . Acesso em 12 fev. 2021.

LIPPI, G.; SANCHIS-GOMAR, F.; HENRY, B. M. Response to: Is newly diagnosed diabetes a stronger risk factor than pre-existing diabetes for COVID-19 severity? Journal of Diabetes, 2020. Disponível em: https://doi.org/10.1111/1753-0407.13127. Acesso em 22 fev. 2021.

LIU, Y.; SUN, W.; LI, J.; CHEN, L.; WANG, Y.; ZHANG, L.; YU, L. Clinical features and progression of acute respiratory distress syndrome in coronavirus disease 2019. Health Sciences Medrxiv, [S.L.], p. 1-28, 20 fev. 2020. Cold Spring Harbor Laboratory. Disponível em: http://dx.doi.org/10.1101/2020.02.17.20024166 . Acesso em 25 fev. 2021.

MACIEL, E. L.; JABOR, P.; GONÇALVES JÚNIOR, E.; TRISTÃO-SÁ, R.; LIMA, L.C.D.; REIS-SANTOS, B.; LIRA, P.; BUSSINGUER, E.C.A.; ZANDONADE, E. Fatores associados ao óbito hospitalar por COVID-19 no Espírito Santo, 2020. Epidemiol. Serv. Saúde, v. 29, n. 4, 2020. Disponível em: https://www.scielosp.org/article/ress/2020.v29n4/e2020413/pt . Acesso em 30 jan. 2021.

MERCÊS, S. O.; LIMA, F. L. O.; NETO, J. R. T. V. Associação da COVID-19 com: idade e comorbidades médicas. Society and Development, v. 9, n.10, e1299108285, 2020. Disponível em: https://www.rsdjournal.org/index.php/rsd/article/view/8285/7478 . Acesso em 30 jan. 2021.

MENDONÇA, F. D.; ROCHA, S.S.; PINHEIRO, D.L.P.; OLIVEIRA, S.V. Região Norte do Brasil e a pandemia de COVID19: análise socioeconômica e epidemiológica. Journal Health Npeps, [S.L.], v. 5, n. 1, p. 20-37, 2020. Universidade do Estado do Mato Grosso - UNEMAT. Disponível em: http://dx.doi.org/10.30681/252610104535. Acesso em 18 fev. 2021.

MINISTÉRIO DA SAÚDE. Sobre a doença. Disponível em: https://coronavirus.saude.gov.br/sobre-a-doenca . Acesso em 08 jan. 2021.

MORAES, E. N.; VIANA, L.G.; RESENDE, L.M.H.; VASCONCELLOS, L.S.; MOURA, A.S.; MENEZES, A.; MANSANO, N.H.; RABELO, R. COVID-19 nas instituições de longa permanência para idosos: estratégias de rastreamento laboratorial e prevenção da propagação da doença. Ciênc. saúde coletiva, v. 25, n. 9, 2020. DOI 10.1590/141381232020259.20382020. Disponível em: https://doi.org/10.1590/1413-81232020259.20382020. Acesso em 06 jan. 2021.

MOREIRA, R. S. COVID-19: unidades de terapia intensiva, ventiladores mecânicos e perfis latentes de mortalidade associados à letalidade no Brasil. Cad. Saúde Pública, v. 36, n.5, :e00080020, 2020. Disponível em: https://doi.org/10.1590/0102-311X00080020 . Acesso em 16 fev. 2021.

NIQUINI, R. P.; LANA, R.M.; PACHECO, A.G.; CRUZ, O.G.; COELHO, F.C.; CARVALHO, L.M.; VILLELA, D.A.M.; GOMES, M.F.C.; BASTOS, L.S. SRAG por COVID-19 no Brasil: descrição e comparação de características demográficas e comorbidades com SRAG por influenza e com a população geral. Cad. Saúde Pública, Rio de Janeiro, v. 26, n. 7, 2020. Disponível em: https://www.scielo.br/scielo.php?script=sci_arttext\&pid=S0102311X2020000705013\&lang=pt Acesso em 23 jan. 2021.

NUNES, B. P.; SOUZA, A.S.S.; NOGUEIRA, J.; ANDRADE, F.B.; THUMÉ, E.; TEIXEIRA, D.S.C.; IMA-COSTA, M.F.; FACCHINI, L.A.; BATISTA, S.R. Multimorbidade e população em risco para COVID-19 grave no Estudo Longitudinal da Saúde dos Idosos Brasileiros. Cad. Saúde Pública, Rio de Janeiro, v. 36, n. 12, 2020. Disponível em: https://www.scielo.br/scielo.php?pid=S0102-311X2020001205003\&script=sci_arttext . Acesso em 25 jan. 2021.

PARK, B. E.; LEE, J.H.; KYOON, P.H.; KIM, H.N.; et al. Impact of Cardiovascular Risk Factors and Cardiovascular Diseases on Outcomes in Patients Hospitalized with COVID-19 in Daegu Metropolitan City. Journal of Korean Medical Science, [S.L.], v. 36, n. 2, p. 1-13, 2021. Korean Academy of Medical Sciences. Disponível em: http://dx.doi.org/10.3346/jkms.2021.36.e15 . Acesso em 04 mar. 2021.

PIANA, A.; COLUCCI, M.E.; VALERIANI, F.; MARCOLONGO, et al. Monitoramento de riscos de transmissão COVID19 por PCR quantitativo em tempo real de rastreamento de gotículas em hospitais e ambientes vivos. ASM 
Journals. e01070-20, 2020. DOI 10.1128 / mSphere.01070-20. Disponível em: https://msphere.asm.org/content/6/1/e01070-20 . Acesso em 08 jan. 2021.

PINNEY, SEAN P.; GIUSTINO, G.; HALPERIN, J.L.; MECHANICK, J.I.; NEIBART, E; OLIN, J.W.; ROSENSON, R.S.; FUSTER, W. Perspectiva histórica do coronavírus, mecanismos da doença e resultados clínicos. J Am Coll Cardiol, v. 76 n.17, p. 1999-2010, 2020. DOI 10.1016 / j. jacc.2020.08.058. Disponível em: https://www.ncbi.nlm.nih.gov/pmc/articles/PMC7571970/ . Acesso em 05 jan. 2021.

RACHE, B.; ROCHA, R.; NUNES, L.; SPINOLA, P.; MALIK, A.M.; MASSUDA, A. Necessidades de infraestrutura do SUS em preparo à COVID-19: leitos de UTI, respiradores e ocupação hospitalar. São Paulo: Instituto de Estudos para Políticas de Saúde, 2020. Disponível em: https://ieps.org.br/wp-content/uploads/2020/04/IEPS-NT3.pdf . Acesso em 18 fev. 2021.

REBOUÇAS, E. R. N.; COSTA, R.F.; MIRANDA, L.R.; CAMPOS, N.G. Perfil demográfico e clínico de pacientes com diagnóstico de COVID-19 em um hospital público de referência na cidade de Fortaleza-Ceará.J. Health Biol Sci., v. 8, n. 1, p. 1-5, 2020. Disponível em: https://periodicos.unichristus.edu.br/jhbs/article/view/3438/1218 . Acesso em: 30 jan. 2021.

SANTOS, P. O. O.; NINOMIYA, V.Y.; CARVALHO, R. T. Envelhecimento e covid-19: o impacto das comorbidades nos idosos e a relação como o novo coronavírus. $2020^{\mathrm{a}}$ Disponível em: https://coronavirus.saude.mg.gov.br/blog/166-envelhecimento-e-covid-19 . Acesso em: 27 jan. 2021.

SANYAOLU, A.; OKORIE, C.; MARINKOVIC, A.; PATIDAR, R.; YOUNIS, K.; DESAI, P.; HOSEIN, Z.; PADDA, I.; MANGAT, J.; ALTAF, M. Comorbidade e seu impacto em pacientes com COVID-19. SN Compr Clin Med, v. 1 n. 8, 2020. DOI 10.1007 / s42399-020-00363-4. Disponível em: https://www.ncbi.nlm.nih.gov/pmc/articles/PMC7314621/\#CR26 . Acesso em: 06 jan. 2021.

SAÚDE, BRASIL. Por que a obesidade é um fator de risco para pessoas com Coronavírus? Disponível em: https://saudebrasil.saude.gov.br/ter-peso-saudavel/por-que-a-obesidade-e-um-fator-de-risco-para-pessoascom-coronavirus. Acesso em 27 jan. 2021.

SHEPPARD, J. P.; NICHOLSON, B.D.; LEE, J.; MACGAGH, D.; et al. Association Between Blood Pressure Control and Coronavirus Disease 2019 Outcomes in 45418 Symptomatic Patients With Hypertension. Hypertension, [S.L.], v. 77, n. 3, p. 846-855, 3, 2021. Ovid Technologies (Wolters Kluwer Health). Disponível em: http://dx.doi.org/10.1161/hypertensionaha.120.16472 . Acesso em 27 fev, 2021.

SOUZA, C. D. F.; LEAL, T. C.; SANTOS, L. G. Doenças do Aparelho Circulatório em Indivíduos com CoVID-19: descrição do perfil clínico e epidemiológico de 197 óbitos. Arquivos Brasileiros de Cardiologia, [S.L.], v. 115, n. 2, p. 281-283, 2020. Sociedade Brasileira de Cardiologia. Disponível em: http://dx.doi.org/10.36660/abc.20200453 . Acesso em 07 mar. 2021.

SULEYMAN, G.; FADEL, R.A.; MALETTE, K.M. Características clínicas e morbidade associadas à doença coronavírus de 2019 em uma série de pacientes na região metropolitana de Detroit. Jama Netw Open., [s. 1], v. 6, n. 3, p. 1-19, 2020. Disponível em: https://doi.org/10.1001/jamanetworkopen.2020.12270. Acesso em 28 fev. 2021.

SVENSSON, P.; HOFMANN, R.; HÄBE,L, H.; JERNBERG, T.; NORDBERG, P. Association between cardiometabolic disease and severe COVID-19: a nationwide case-control study of patients requiring invasive mechanical ventilation. BMJ Open. $\mathrm{v}$. 11, e044486, 2021. Disponível em: https://doi.org/10.1136/bmjopen-2020-044486 . Acesso em 15 mar. 2021.

TAJI, L.; THOMAS, D.; OLIVER, M.J.; IP, J.; TANG, Y.; YEUNG, A.; COOPER, R.; HOUSE, A.A.; MCFARLANE, P.; BLAKE, P.G. COVID-19 in patients undergoing long-term dialysis in Ontario. Canadian Medical Association Journal, [S.L.], v. 193, n. 8, p. 278-284, 4 fev. 2021. Joule Inc. Disponível em: http://dx.doi.org/10.1503/cmaj.202601 . Acesso em 05 mar. 2021.

TALEGHANI, N., TAGHIPOUR, F. Diagnóstico de COVID-19 para controle da pandemia: uma revisão do estado da arte. Biosens Bioelectron, v. 174, 2020. Disponível em: https://www.ncbi.nlm.nih.gov/pmc/articles/PMC7694563/ . Acesso em: 26 jan. 2021. 
TEIXEIRA, D. B. S. Atenção à saúde do homem: análise da sua resistência na procura dos serviços de saúde. Rev Cubana Enferm [Internet] 2016 [acessado em 18 ago. 2020]; v. 32, n. 4. Disponível em: http://revenfermeria.sld. cu/index.php/enf/article/view/985. Acesso em 07 mar. 2021.

TEIXEIRA, C. F. S.; SOARES, C.M.; SOUZA, E.A.; LISBOA, E.S.; PINTO, I.C.M.; ANDRADE, L.R.; ESPIRIDÃO, M.A. A saúde dos profissionais de saúde no enfrentamento da pandemia de Covid-19. Ciênc. saúde coletiva, Rio de Janeiro, v. 25, n. 9, Dec. 2020. Disponível em: http://dx.doi.org/10.1590/1413-81232020259.19562020 . Acesso em 26 jan. 2021.

TOLIA, V. M.; CHAN, T. C.; CASTILLO, E. M. Preliminary results of initial testing for coronavirus (COVID-19) in the emergency department. West J Emerg Med [Internet]. 2020 Mar [cited 2020 Aug 29]; v. 21, n. 3, p. 503-6. Disponível em: https://doi.org/10.5811/westjem.2020.3.47348 . Acesso em 15 mar. 2021.

WANG, W.; ZHAO, X.; WEI, W.; FAN, W.; GAO, K.; HE, S.; ZHUANG, X. Os inibidores da enzima de conversão da angiotensina (IECA) ou bloqueadores do receptor da angiotensina (ARA) podem ser seguros para pacientes com COVID-19. BMC Infect Dis v. 21, n. 114, 2021. Disponível em: https://doi.org/10.1186/s12879-021-05821-5 . Acesso em 17 fev. 2021.

WORLD HEALTH ORGANIZATION - WHO. Coronavirus disease 2019 (COVID-19) - WHO Coronavirus Disease (COVID-19) dashboard [Internet]. Geneve: WHO; 2020. Disponível em: https://covid19.who.int Acesso em 20 fev. 2021.

YANG, J.; ZHENG, Y.; GOU, X.; PU, K.; CHEN, Z.; GOU, Q.; JI, R.; WNAG, H.; WANG, Y.; ZHOU, Y. Prevalence of comorbidities and its effects in patients infected with SARS-CoV-2: a systematic review and meta-analysis. International Journal of Infectious Diseases, [S.L.], v. 94, p. 91-95, maio 2020. Elsevier BV. Disponível em: http://dx.doi.org/10.1016/j.ijid.2020.03.017 . Acesso em 23 fev. 2021.

YEHIA, B. R.; WINEGAR, A.; FOGEL, R.; FAKIH, M.; OTTENBACHER, A.; JESSE, C.; BUFALINO, A.; HUANG, R.; CACCHIONE, J. Associação de raça com mortalidade entre pacientes hospitalizados com doença por coronavírus 2019 (COVID-19) em 92 hospitais dos EUA. JAMA Netw Open, v. 3, n. 8, e2018039, 2020. Disponível em: https://www.ncbi.nlm.nih.gov/pmc/articles/PMC7435340/\#zoi200646r4 . Acesso em 28 jan. 2021.

ZHANG, H.; PENNINGER, J.M.; LI, Y.; ZHONG, N.; SLUTSKY, A.S. Enzima conversora de angiotensina 2 (ACE2) como um receptor SARS-CoV-2: mecanismos moleculares e potencial alvo terapêutico. Intensive Care Med, v. 46, n. 4, p. 586-90, 2020. Disponível em: https://www.ncbi.nlm.nih.gov/pmc/articles/PMC7079879/. Acesso em 01 fev. 2021.

ZHAO, J.; YUAN, Q.; WANG, H.; LIU, W.; et al. Antibody Responses to SARS-CoV-2 in Patients With Novel Coronavirus Disease 2019. Clinical Infectious Diseases, [S.L.], v. 71, n. 16, p. 2027-2034, 28 maio 2020. Oxford University Press (OUP). Disponível em: http://dx.doi.org/10.1093/cid/ciaa344 . Acesso em 15 fev. 2021.

ZHOU, M.; DONG, C.; LI, C.; WANG, Y.; LIAO, H.; SHI, H.; LI, A.P.; WNAG, J.; HU, Y; ZHENG, C. Longitudinal changes in COVID-19 clinical measures and correlation with the extent of CT lung abnormalities. International Journal of Medical Sciences, [S.L.], v. 18, n. 5, p. 1277-1284, 2021. Ivyspring International Publisher. Disponível em: http://dx.doi.org/10.7150/ijms.51279 . Acesso em 05 mar. 2021.

\section{(c)) EY}

Este trabalho está licenciado com uma Licença Creative Commons - Atribuição 4.0 Internacional. 